\title{
Circulatory Hsa_Circ_0007777 Expression Level as A Predictive Biomarker of Thyroid Dysfunction in Patients with Hashimoto's Thyroiditis
}

\author{
Nearmeen M. Rashad ${ }^{1}$, Marwa H.S Hussien ${ }^{2}$, Rehab M. Atef ${ }^{3}$, Shimaa Abdelmoneem ${ }^{1}$, Mohamed G. Hamed ${ }^{1}$ \\ Departments of ${ }^{1}$ Internal Medicine, ${ }^{2}$ Medical Biochemistry and \\ ${ }^{3}$ Clinical Pathology, Faculty of Medicine, Zagazig University, Egypt \\ *Corresponding author: Mohamed G. Hamed, Mobile: (+20) 01228588047, E-mail: mohamed1gaber@gmail.com
}

\begin{abstract}
Background: Hashimoto's thyroiditis (HT) is the most common cause of hypothyroidism in iodine-sufficient areas of the world.The pathophysiology of HT is contributed to a combination of genetic susceptibility and environmental factors.

Objective: We aimed in the current study to investigate the relative expression level of hsa_circ_0007777 in patients with or without HT and to assess their associations with thyroid dysfunction.

Patients and Methods: A case-control study included 70 patients with HT and 70 healthy control subjects.Antithyroglobulin (anti-TG), anti-thyroid peroxidase (anti-TPO) were measured. Real-Time Quantitative PCR (RT-qPCR) analyses were used to detect the relative expression levels of hsa_circ_0007777.

Results: Hsa_circ_0007777 relative expression level was up regulated in patients with HT in particular patients with clinical hypothyroidism (CHT) and subclinical hypothyroidism (SCHT) compared to the euthyroid group. Andit was correlated positively with TSH, anti-TPO, and anti-TG. Moreover, linear regression analyses test revealed that in HT patients the main independent variables associated with hsa_circ_0007777 relative expression level were anti-TPO and anti-TG.

Conclusion: The relative expression levels of hsa_circ_0007777 were significantly increased in patients with HT more specifically in patients with clinical and subclinical hypothyroidism.
\end{abstract}

Keywords: hsa_circ_0007777, HT; RT-qPCR, Gene expression, Anti-TPO.

\section{INTRODUCTION}

Hashimoto's thyroiditis (HT), which is characterized by thyroid-specific autoantibodies, is one of the most common autoimmune diseases. Although the exact origin of Hashimoto's thyroiditis is unknown, it is believed to be caused by a mix of inherited, environmental, and epigenetic factors. It is also known as chronic lymphocytic thyroiditis ${ }^{(\mathbf{1})}$. Although HT can induce overt hypothyroidism, the majority of patients with high TSH and normal thyroid hormone levels have subclinical hypothyroidism (2). Several important genetics and environmental variables that contribute to the pathogenesis of HT have been studied in depth ${ }^{(3)}$. Despite this, little is known about the epigenetic anomalies that occur in HT, particularly those that involve circular RNA (circRNA). According to accumulating evidence, non-genetic factors control gene expression and phenotypes, as well as their involvement in disease development ${ }^{(4)}$. As a result, epigenetic processes can affect genes involved in the immune system or thyroid, and epigenetic dysfunctions of these genes can result in autoimmune diseases ${ }^{(5)}$.

CircRNAs are single-stranded closed circular RNA molecules that lack poly (A) tails and $5^{\prime}-3^{\prime}$ ends (1). CircRNAs were discovered in eukaryotes in 1979 as an endogenous RNA splicing product and in humans in 1986 after hepatitis delta virus infection ${ }^{(3,4)}$. CircRNAs have been related to the pathophysiology of a variety of human illnesses, including osteoarthritis (5) and diabetes ${ }^{(6)}$. CircRNAs have been found to play a key function in autoimmunity among epigenetic regulators (4). According to new findings, circRNAs perform complex activities such as serving as endogenous RNAs for sponge miRNAs and controlling parental gene expression. Furthermore, through suppressing miR-145 expression, circRNAs are responsible for the regulation of vascular smooth muscle cells (VSMCs) migration, proliferation, and differentiation ${ }^{(7)}$.

Furthermore, Li et $\boldsymbol{a l} .{ }^{\left({ }^{(8)}\right.}$ detected dysregulation of hsa_circ_0045272expression level in systemic lupus erythematosus.

We aimed in the current study to investigate the relative expression level of hsa_circ_0007777 in patients with or without HT and to assess their associations with thyroid dysfunction.

\section{PATIENTS AND METHODS}

A case-control study included 140 unrelated women: 70 patients with HT and 70 healthy controls. The enrolled HT patients were stratified into 5 subgroups based on the guidelines for the use of thyroid function tests, all patients were subjected to thorough history taking and full clinical assessment. Patients with a history of cancer, liver, kidney or any active inflammatory diseases were excluded from this study.

All the participants had the following laboratory investigation, thyroid function tests including FT3, FT4, anti-thyroglobulin antibodies (antiTG), anti-thyroid peroxidase antibodies (anti-TPO) and serum thyroid-stimulating hormone (TSH), FT3, and FT4 concentrations were measured by luminescencebased immunoassay using Immulite 2000 (Diagnostic Products Corp., Los Angeles, California, USA) ${ }^{(10)}$. 
The reference ranges for TSH, FT3, and FT4 were $0.4-4.0 \mathrm{mU} / \mathrm{L}, 3.5-5.5 \mathrm{pmol} / \mathrm{L}$, and $10.0-26.0$ $\mathrm{pmol} / \mathrm{L}$, respectively ${ }^{(11)}$. Serum TPOAb and TG Ab were measured by ELISA using diagnostica $\mathrm{GmbH}$, Carl-Zeiss-Strasse; 2010-2013, and Genesis Diagnostics, Littleport, UK; 2014-2015, respectively. The levels of antibodies were considered positive if they were $>100 \mathrm{IU} / \mathrm{ml}$ for anti-TG and $>75 \mathrm{IU} / \mathrm{ml}$ for anti TPO. Positivity of at least one antibody was considered as having autoimmunity of thyroid ${ }^{(\mathbf{1 2})}$.

\section{Extraction of RNA}

Total RNA extraction kit was used to get total RNA. (Cat. No. BSC52S1); supplied by Bioer Technology Co., China. HiSenScript is an RNA reverse transcription kit. (Cat. No. 25014); supplied by iNtRON Biotechnology, Inc., South Korea. It was employed in the production of cDNA from total RNA. The RNA quality was quantified by A260 using a $\mathrm{UV} /$ spectrophotometer.

Total RNA obtained from PBMCs with Trizol (Invitrogen, Carlsbad, CA USA) was used for synthesizing cDNAs with the Rever TraAcreal-time qPCR kit (Toyobo, Osaka, Japan). First-strand cDNA $(2 \mathrm{~mL})$ was used for PCR performed in triplicate, with SYBR Green SuperMix (Bio-Rad, Hercules, CA, USA). b- Actin was applied as an internal reference for circRNAs to avoid potential aberrance in concentration and efficiency of transcription.

Amplification protocol consisted of initial denaturation with polymerase activation at $95^{\circ} \mathrm{C}$ for 15 min, then 40 cycles of denaturation at $94^{\circ} \mathrm{C}$ for $15 \mathrm{sec}$, annealing at $55^{\circ} \mathrm{C}$ for $30 \mathrm{sec}$ and extension at $70^{\circ} \mathrm{C}$ for $30 \mathrm{sec}$. Expression levels of each gene were normalized to $\beta$-actin. Relative gene expression of hsa_circ_0007777 was analyzed and calculated using RT-PCR and was measured by the $2^{-\Delta C t}$ method.

\section{Ethical consent}

An approval of the study was obtained from Zagazig University Academic and Ethical Committee. Every patient signed an informed written consent for acceptance of the study. This work has been carried out in accordance with The Code of Ethics of the World Medical Association (Declaration of Helsinki) for studies involving humans.

\section{Statistical analysis}

Analysis of data was performed using SPSS v.26. The data were expressed using descriptive statistics (mean \pm standard deviation) and were analyzed using the " $\mathrm{t}$ " test. The correlation between the relative expression level of hsa_circ_0007777 and other studied parameter was assessed by Pearson correlation test.

We tested the association between the relative expression level of hsa_circ_0007777 and other studied variables in patients with T2DM and detected the independent variables by linear regression. Receiver operating characteristics (ROC) tested the diagnostic powers of hsa_circ_0007777 levels among studied subjects.

\section{RESULTS}

According to our results, 13 males and 57 females were enrolled in the control group and 10 males, as well as 60 females, were registered in the case group (HT group). In the patient with HT, there were significantly higher values of age, systolic and diastolic blood pressure, TSH, anti-TG, and anti-TPO compared to the control group. On the contrary, FT3 and FT4 levels were significantly lower in the HT group as compared to the control group $(\mathrm{P}<0.001)$ (Table 1$)$.

Table (1): Clinical and laboratory characteristics in studied groups.

\begin{tabular}{|l|c|c|c|}
\hline Parameter & $\begin{array}{c}\text { Healthy controls } \\
\text { group }(\boldsymbol{n}=\mathbf{7 0})\end{array}$ & $\begin{array}{c}\text { Patient with HT } \\
\text { group }(\boldsymbol{n}=\mathbf{7 0})\end{array}$ & P value \\
\hline Age $($ years) & $37.4 \pm 2.51$ & $42.9 \pm 2.36$ & $<0.001^{*}$ \\
\hline Systolic blood pressure $(\mathrm{mmHg})$ & $125.34 \pm 7.25$ & $137.52 \pm 7.07$ & $<0.001^{*}$ \\
\hline Diastolic blood pressure $(\mathrm{mmHg})$ & $82.11 \pm 4.7$ & $85.6 \pm 6.52$ & $<0.001^{*}$ \\
\hline FT3 $(\mathrm{pg} / \mathrm{ml})$ & $2.3 \pm 0.2$ & $1.3 \pm 0.51$ & $<0.001^{*}$ \\
\hline FT4 $(\mathrm{ng} / \mathrm{dl})$ & $1.45 \pm 0.1$ & $0.67 \pm 0.1$ & $<0.001^{*}$ \\
\hline TSH $(\mu \mathrm{IU} / \mathrm{ml})$ & $2.22 \pm 1.87$ & $26.3 \pm 13.9$ & $<0.001^{*}$ \\
\hline Anti TPO $(\mathrm{IU} / \mathrm{ml})$ & $33.64 \pm 1.62$ & $259.64 \pm 54.11$ & $<0.001^{*}$ \\
\hline AntiTG $(\mathrm{IU} / \mathrm{ml})$ & $22.99 \pm 1.46$ & $150.36 \pm 19.16$ & $<0.001^{*}$ \\
\hline
\end{tabular}

Anti-TG; anti-thyroglobulin antibodies, Anti-TPO; anti-thyroid peroxidase antibodies, TSH; serum thyroid-stimulating hormone, $* \mathrm{P}<0.05$ when compared with control group.

HT groups were classified into 5 groups according to their thyroid state: SCHT (n=22), CHT (n=19), subclinical hyperthyroidism $(n=4)$, hyperthyroidism $(n=5)$ and euthyroid $(n=30)$. There were statistically significant differences between these groups as regards FT3, FT4, and TSH $(\mathrm{P}<0.001)$ as shown in table $(2)$. 
Table (2): Clinical and laboratory characteristics in HT patients according to their thyroid state.

\begin{tabular}{|l|c|c|c|c|c|}
\hline Parameters & $\begin{array}{c}\text { SCHT } \\
(\mathbf{n = 2 2})\end{array}$ & $\begin{array}{c}\text { CHT } \\
(\mathbf{n = 1 9})\end{array}$ & $\begin{array}{c}\text { Subclinical } \\
\text { hyperthyroidism } \\
(\boldsymbol{n}=\mathbf{4})\end{array}$ & $\begin{array}{c}\text { Hyperthyroidism } \\
(\boldsymbol{n = 5})\end{array}$ & $\begin{array}{c}\text { Euthyroid } \\
(\mathbf{n = 3 0})\end{array}$ \\
\hline FT3(pg/ml) & $2.81 \pm 0.42$ & $0.82 \pm 0.2^{*}$ & $2.34 \pm 0.37$ & $11.01 \pm 2.64^{*}$ & $3.11 \pm 0.34$ \\
\hline FT4 $(\mathrm{ng} / \mathrm{dl})$ & $3.1 \pm 0.92$ & $0.72 \pm 0.021^{*}$ & $1.64 \pm 0.903$ & $5.22 \pm 1.45^{*}$ & $1.32 \pm 0.34$ \\
\hline TSH $(\mu \mathrm{IU} / \mathrm{ml})$ & $8.62 \pm 1.56^{*}$ & $38.48 \pm 182^{*}$ & $0.113 \pm 0.01^{*}$ & $0.07 \pm 0.01^{*}$ & $2.5 \pm 0.97$ \\
\hline $\begin{array}{l}\text { Anti } \\
\text { TPO }(\mathrm{IU} / \mathrm{ml})\end{array}$ & $177.32 \pm 31.3$ & $185.2 \pm 12.22^{*}$ & $153.1 \pm 12.52$ & $150.4 \pm 13.03$ & $58.1 \pm 9.6$ \\
\hline AntiTG $(\mathrm{IU} / \mathrm{ml})$ & $92.49 \pm 23.13$ & $115.75 \pm 13.46$ & $102.76 \pm 11.1$ & $111.3 \pm 24.68$ & $31.87 \pm 6.6$ \\
\hline
\end{tabular}

hypothyroidism; CHT, clinical hypothyroidism; TSH, thyroid stimulating hormone; FT3 free triidothyronine, FT4; free thyroxine ,

Anti TG ; anti thyroglobulin antibodies, anti-TPO; anti-thyroid peroxidase antibodies $\quad * \mathrm{P}<0.001$ when compared to euthyroid group.

There was a significantly higher level of hsa_circ_0007777 relative expression in the HT group (3.88 \pm 1.025$)$ compared to the control group $(1.69 \pm 0.69) \mathrm{P}<0.001$ (Figure 1).

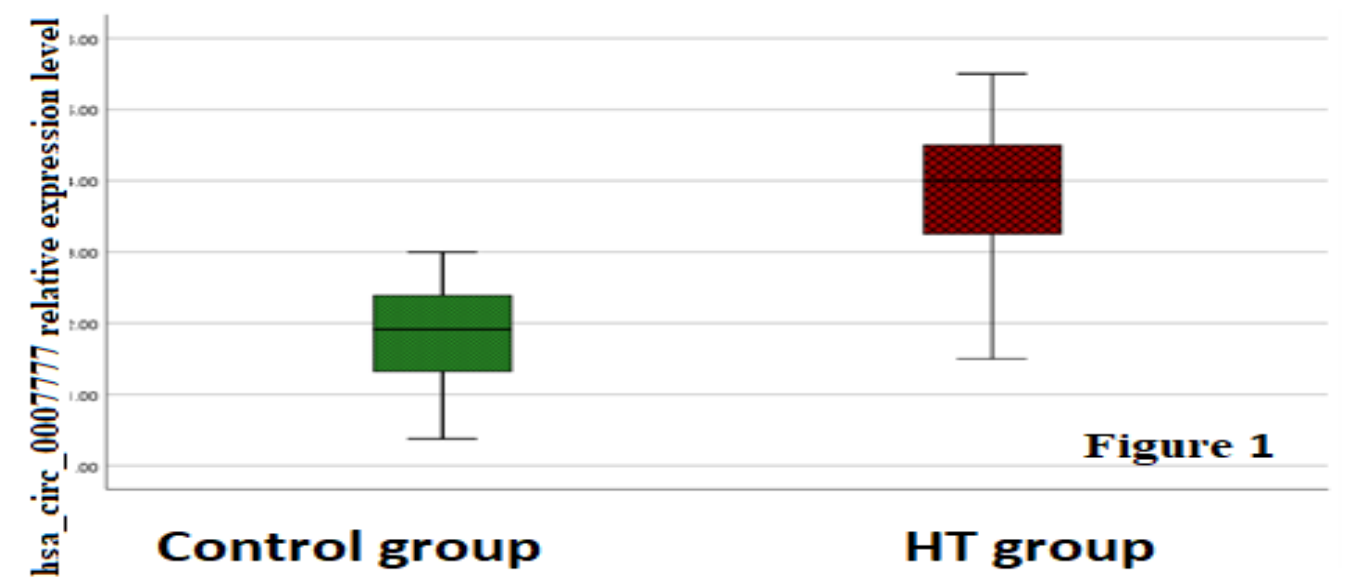

Figure (1): Comparison of hsa_circ_0007777 relative expression level in studied groups.

Interestingly, there was a significant difference between studied subgroups as regards hsa_circ_0007777 relative expression as the levels were SCHT $(4.3 \pm 0.578)$, CHT $(4.46 \pm 0.802)$, subclinical hyperthyroidism $(3.98 \pm 0.72)$, hyperthyroidism $(3.6 \pm 1.07)$, and euthyroid $(1.71 \pm 0.712) \mathrm{P}<0.001$ (Figure 2 ).

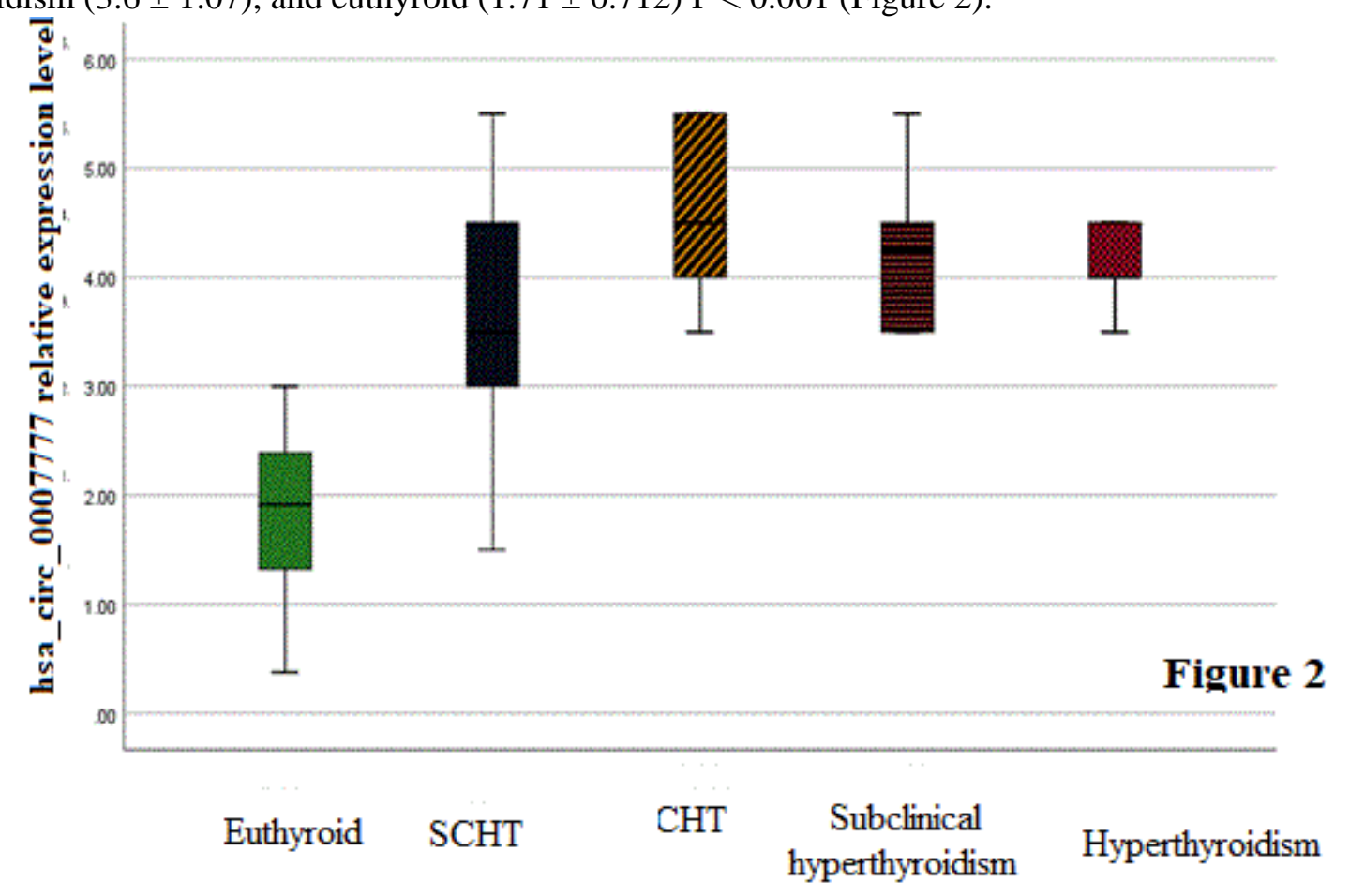

Figure (2): Comparison of hsa_circ_0007777 relative expression level in HT subgroups according to thyroid function. In the HT group, hsa_circ_0007777 relative expression levels were significantly positively correlated with TSH, antiTPO, and anti-TG. On the contrary, there were significant negative correlations between hsa_circ_0007777 relative expression levels and FT3 and FT4 P $<0.001$ (Table 3). 
Table (3): Pearson correlations of hsa_circ_0007777 relative expression level with clinical and laboratory characteristics in HT patients.

\begin{tabular}{|l|c|c|}
\hline \multicolumn{1}{|c|}{ Characteristics } & \multicolumn{2}{|c|}{$\begin{array}{c}\text { HT group } \\
(\mathbf{n = 7 0 )}\end{array}$} \\
\cline { 2 - 3 } & R & $\boldsymbol{P}$ \\
\hline FT3 $(\mathrm{pg} / \mathrm{ml})$ & -0.567 & $<0.001$ \\
\hline FT4 $(\mathrm{ng} / \mathrm{dl})$ & -0.672 & $<0.001$ \\
\hline TSH $(\mu \mathrm{IU} / \mathrm{ml})$ & 0.986 & $<0.001$ \\
\hline Anti TPO $(\mathrm{IU} / \mathrm{ml})$ & 0.831 & $<0.001$ \\
\hline AntiTG(IU/ml) & 0.831 & $<0.001$ \\
\hline
\end{tabular}

In HT $(n=70)$, linear regression analysis showed that hsa_circ_0007777 relative expression level was independently correlated with anti-TPO and anti-TGP $<0.001$ (Table 4).

Table (4): Linear regression analyses in HT patients to test the influences of the main independent variables against hsa_circ_0007777 relative expression level (dependent variable).

\begin{tabular}{|l|c|c|c|c|c|c|c|}
\hline \multirow{2}{*}{ Model } & \multicolumn{2}{|c|}{$\begin{array}{c}\text { Unstandardized } \\
\text { Coefficients }\end{array}$} & $\begin{array}{c}\text { Standardized } \\
\text { Coefficients }\end{array}$ & \multirow{2}{*}{ t } & \multicolumn{2}{c|}{ P } & \multicolumn{2}{c|}{$95 \%$ C.I } \\
\cline { 2 - 6 } & B & S E & Beta & & & $\begin{array}{c}\text { Lower } \\
\text { Bound }\end{array}$ & $\begin{array}{c}\text { Upper } \\
\text { Bound }\end{array}$ \\
\hline Constant & 5.919 & .449 & & 13.169 & $<0.001$ & 5.030 & 6.808 \\
\hline FT3 $(\mathrm{pg} / \mathrm{ml})$ & 2.302 & 0.666 & 0.237 & 1.382 & 0.169 & -0.993 & 5.596 \\
\hline FT4(ng/dl) & -0.095 & 0.028 & $0-.109$ & -0.742 & 0.460 & $0-.348$ & 0.158 \\
\hline TSH $(\mu \mathrm{IU} / \mathrm{ml})$ & 0.004 & 0.001 & 0.033 & 0.385 & 0.701 & -0.015 & 0.023 \\
\hline Anti TPO $(\mathrm{IU} / \mathrm{ml})$ & -1.593 & 0.251 & -0.457 & -6.342 & $<0.001$ & -2.090 & -1.096 \\
\hline AntiTG(IU/ml) & -0.006 & 0.002 & -0.277 & -3.845 & $<0.001$ & -0.010 & -0.003 \\
\hline
\end{tabular}

We further investigated the potential diagnostic value of hsa_circ_0007777 relative expression level by ROC curves for diagnosis of HT, the cutoff values were 2.443 and the AUC was 0.939 (95\% CI $=0.893-0.979)$. Additionally, the sensitivities and the specificities of hsa_circ_0007777 were $87.1 \%$ and $80 \%$ respectively P $<0.001$ (Figure 3).

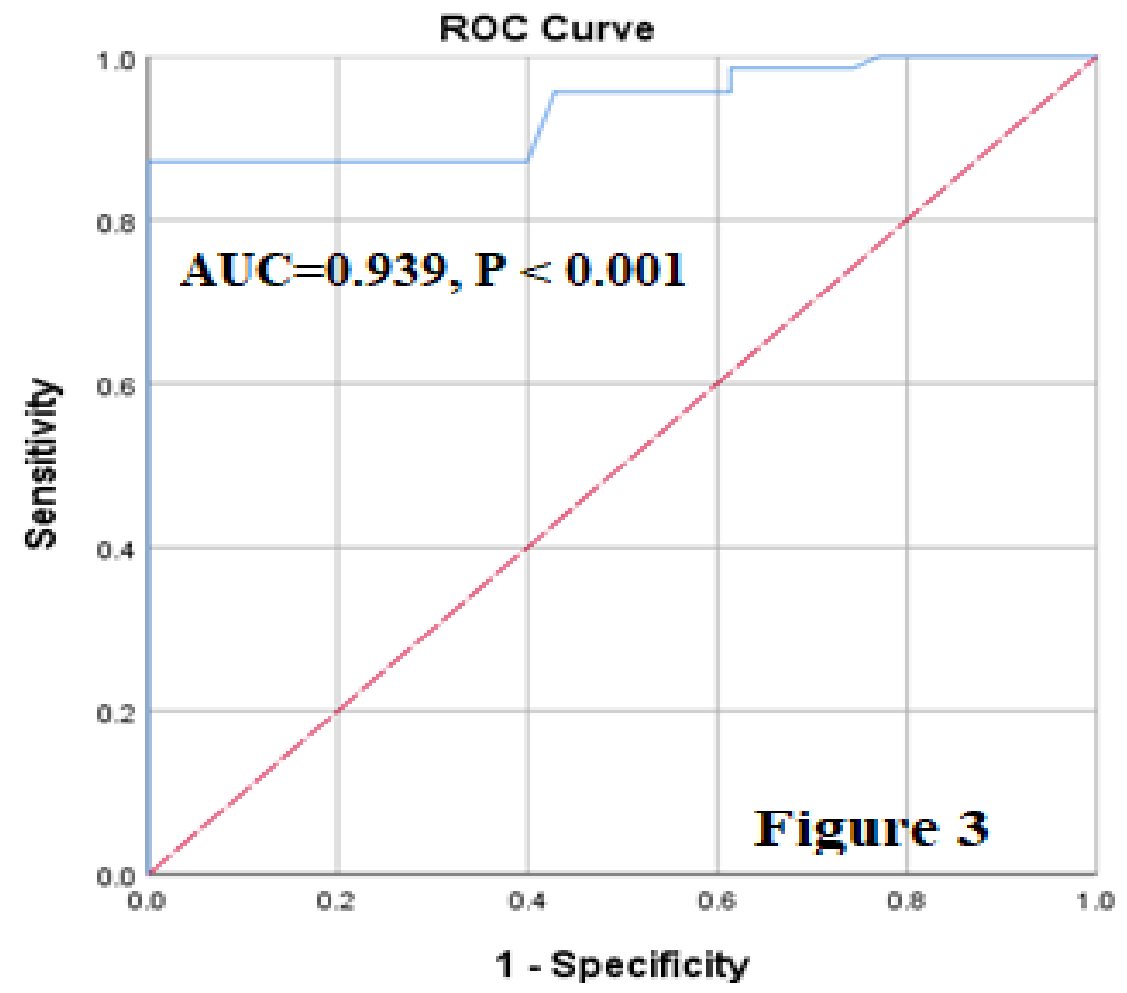

Figure (3): The accuracy of hsa_circ_0007777 relative expression level for diagnosis of HT by ROC analysis. 


\section{DISCUSSION}

There was growing evidence that immunogenetics has a major role in HT pathogenesis. Consequently, a hypothesis could be formulated that polymorphism in these immune-modulating genes can dysregulate immune tolerance and alter $\mathrm{T}$ cells' interactions ${ }^{(\mathbf{1 3})}$. There was a lot of evidence that raised thyroid autoantibodies, including TPOAb and thyroid globulin antibody ( $\mathrm{TgAb})$, may present in a diseasefree population. There was a lot of evidence emphasizing the predictive role of positive thyroid autoantibodies in thyroid dysfunction (14). Additionally, it is widely known that the level of thyroid autoantibodies varies between populations and may be influenced by heredity, environment, and other factors for example birth weight, iodine excess and deficiency and parity ${ }^{(15)}$.

A preponderance of evidence suggests that young patients often have lower titers of TPOAb, and even occasional negative levels, despite the clinically proven disease. Intriguing reports are investigating the relation between thyroid sonography of HT and antiTPO levels. They observed that ultrasound patterns suggestive of HT may also be found in about $20 \%$ of individuals with negative TPOAb concentration (16). Subsequently, we aimed in the current study to investigate the relative expression level of hsa_circ_0007777 as a predictive genetic marker of HT and to assess their associations with thyroid dysfunction. To our knowledge, this is the first report demonstrating the correlation between hsa_circ_0007777 expression and thyroid dysfunction among Egyptian patients with HT.

The results of our research demonstrated that the prevalence of thyroid dysfunction among patients with HT groups was that 22 patients had SCHT, 19 had CHT, four patients had subclinical hyperthyroidism, 5 patients had hyperthyroidism and 30 patients were euthyroid. The interesting result of our study was that level of hsa_circ_0007777 relative expression in the HT group was significantly higher compared to the control. Intriguingly, there was a significant difference between studied subgroups as regard hsa_circ_0007777 relative expression as the levels were significantly higher in particular patients with CHT and SCHT compared to the euthyroid group. In agreement with our study, Xiong $\boldsymbol{e t} \boldsymbol{a l} .{ }^{(17)}$ detected upregulation of hsa_circ_0007777 relative expression levels in HT patients. This study investigated 627 differentially expressed circRNAs and they found that 370 circRNAs expression levels were upregulated and 257circRNAs expression levels were downregulated, in PBMCs of patients with HT. CircRNAs have been proposed as potential biomarkers and contributors to the pathophysiology of autoimmune disorders such as systemic lupus erythematosus ${ }^{(\mathbf{1 8})}$. It may be assumed that hsa_circ_0001859 regulates the expression of
ATF2 by sponging miR204/211, which promotes inflammation in SW982 cells ${ }^{(\mathbf{1 9})}$.

The results of our research demonstrated that in the HT group, hsa_circ_0007777 relative expression levels were significantly positively correlated with TSH, anti-TPO, and anti-TG. On the contrary, there were significant negative correlations between hsa_circ_0007777 relative expression levels and FT3 and FT4. In addition, linear regression analyses test revealed that in HT patients the main independent variables anti-TPO and anti-TG. According to reports of Xiong et $\boldsymbol{a l} .{ }^{(17)}$, there was a positive correlation between expression of hsa_circ_0089172 and TPO Ab, although the correlation between the expression of hsa_circ_0089172 and TGAb had no statistical significance result. Zhao et al. ${ }^{(20)}$ study found a positive correlation between the expression of PD1+ T follicular helper (Tfh) cells and TGAb, and Tfh17 cell proportions correlate positively with the expression of TGAb and TPOAb.

Even more importantly, we further investigated the potential diagnostic value of hsa_circ_0007777 relative expression level by ROC curves for diagnosis of HT and we found that the sensitivities and the specificities of hsa_circ_0007777 were $87.1 \%$ and $80 \%$ respectively. In agreement with our study, Xiong et al. ${ }^{(\mathbf{1 7 )}}$ detected that the sensitivities and the specificities of hsa_circ_0007777 relative expression levels were $79.1 \%$ and $38.1 \%$ respectively. There is a lot of evidence highlighting the connection between circRNAs and HT. Also they showed that upregulated circRNAs had great significance in the pathogenesis of HT ${ }^{(21)}$.

\section{CONCLUSION}

Hsa_circ_0007777 relative expression level was upregulated in patients with HT in particular patients with CHT and SCHT compared to the euthyroid group, and correlated positively with TSH, anti-TPO, and anti-TG. Moreover, linear regression analyses test revealed that in HT patients, the main independent variables anti-TPO and anti-TG. Receiver operating characteristic (ROC) curve analysis suggested that hsa_circ_0007777 relative expression level had significant value in HT diagnosis. Taken together, these results demonstrated that hsa_circ_0007777 is a potential diagnostic biomarker of HT.

\section{REFERENCES}

1. Ralli M, Angeletti D, Fiore $M$ et al. (2020): Hashimoto's thyroiditis: An update on pathogenic mechanisms, diagnostic protocols, therapeutic strategies, and potential malignant transformation. Autoimmun Rev., 19 (10): 102649.

2. Fröhlich E, Wahl R (2017): Thyroid Autoimmunity: Role of Anti-thyroid Antibodies in Thyroid and ExtraThyroidal Diseases. Front Immunol., 8: 521-26. 
3. Tomer Y (2014): Mechanisms of autoimmune thyroid diseases: from genetics to epigenetics. Annu Rev Pathol., 9: 147-56.

4. Canas C, Canas F, Bonilla-Abadia F et al. (2016): Epigenetics changes associated to environmental triggers in autoimmunity. Autoimmunity, 49 (1): 1-11.

5. Liu Q, Zhang X, Hu X et al. (2016): Circular RNA related to the chondrocyte ECM regulates MMP13 expression by functioning as a MiR-136 'Sponge' in human cartilage degradation. Sci Rep., 6: 22572-76.

6. Zhao Z, Li X, Jian D et al. (2017): Hsa_circ_0054633 in peripheral blood can be used as a diagnostic biomarker of pre-diabetes and type 2 diabetes mellitus. Acta Diabetol., 54: 237-45.

7. Zhang Y, Liang W, Zhang $\mathbf{P}$ et al. (2017): Circular RNAs: emerging cancer biomarkers and targets. J Exp Clin Cancer Res., 36: 152-156.

8. Hall I, Climent-Salarich M, Quintavalle $M$ et al. (2019): Circ_Lrp6, a Circular RNA Enriched in Vascular Smooth Muscle Cells, Acts as a Sponge Regulating miRNA-145 Function. Circ Res., 124: 498510.

9. Li L, Zhu Z, Zhao W et al. (2018): Circular RNA expression profile and potential function of hsa_circ_0045272 in systemic lupus erythematosus. Immunology, 155: 137-149.

10. Sánchez-Carbayo $M$, Mauri $M$, Alfayate $R$ et al. (1999): Analytical and clinical evaluation of TSH and thyroid hormones by electrochemiluminescent immunoassays. Clin Biochem., 32: 395-403.

11. Metwalley K, Farghaly H, Saad K et al. (2016): Oxidative status in children and adolescents with autoimmune thyroiditis. Clin Exp Med., 16: 571-5.

12. Brown $R$ (2013): Autoimmune thyroiditis in childhood. J Clin Res Pediatr Endocrinol., 1: 45-49.
13. Lee H, Li C, Hammerstad $\mathrm{S}$ et al. (2015): Immunogenetics of autoimmune thyroid diseases: a comprehensive review. J Autoimmun., 64: 82-90.

14. Yan Y, Gao X, Zeng J et al. (2015): The association between thyroid autoantibodies in serum and abnormal function and structure of the thyroid. J Int Med Res., 43 (3): 412-423.

15. Brix T, Hansen P, Kyvik K et al. (2004): Aggregation of thyroid autoantibodies in first-degree relatives of patients with autoimmune thyroid disease is mainly due to genes: a twin study. Clin Endocrinol (Oxf), 60 (3): 329-334.

16. Biondi B, Cooper D (2008): The clinical significance of subclinical thyroid dysfunction. Endocrine Reviews, 29: 76-131.

17. Xiong S, Peng H, Ding $X$ et al. (2019): Circular RNA Expression Profiling and the Potential Role of hsa_circ_0089172 in Hashimoto's Thyroiditis via Sponging miR125a-3p. Mol Ther Nucleic Acids, 17: $38-48$.

18. Zhang M, Wang J, Zhu Z et al. (2018): Differentially expressed circular RNAs in systemic lupus erythematosus and their clinical significance. Biomed Pharmacother., 107: 1720-1727.

19. Li B, Li N, Zhang L et al. (2018): Hsa_circ_0001859 Regulates ATF2 Expression by Functioning as an MiR-204/211 Sponge in Human Rheumatoid Arthritis. J Immunol Res., 17: 9412387.

20. Zhao J, Chen Y, Zhao $Q$ et al. (2018): Increased circulating Tfh17 and PD-1+Tfh cells are associated with autoantibodies in Hashimoto's thyroiditis. Autoimmunity, 51: 352-359.

21. Koc A, Batar B, Celik O et al. (2014): Polymorphism of the NFKB1 affects the serum inflammatory levels of IL-6 in Hashimoto thyroiditis in a Turkish population. Immunobiology, 219: 531-536. 